\title{
MARKETING DE INFORMACIÓN EN EMPRESAS DE FRANQUICIA
}

\section{Juan Monserrat-Gauchi y María-Carmen Quiles-Soler}

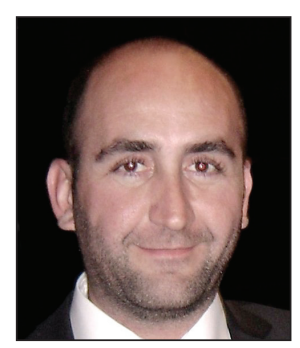

Juan Monserrat-Gauchi es doctor en ciencias de la información por la Universidad Cardenal Herrera CEU de Valencia y licenciado en ciencias de la información: publicidad y relaciones públicas por la Universidad Politécnica de Valencia. Ha realizado estancias de investigación en: Valparaíso, Chile, Universidad Nacional Autónoma de México y The California State University, EUA. Sus principales líneas de investigación son la comunicación en la empresa de franquicia, sistemas y procesos de comunicación comercial, y comunicación para públicos específicos. Pertenece a los grupos de investigación Compubes y Fisec, y es miembro del Observatorio de Revistas Científicas de Ciencias Sociales. Ha sido Premio Nacional de Franquicia al mejor trabajo de análisis e investigación. http://orcid.org/0000-0003-1818-2968

juan.monserrat@ua.es

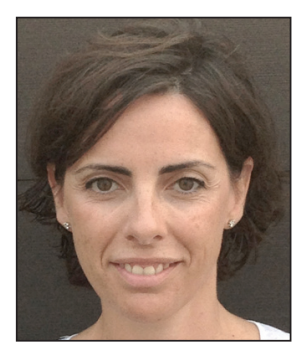

María-Carmen Quiles-Soler es licenciada en ciencias de la información (rama de publicidad y relaciones públicas) por la Universidad Complutense de Madrid y doctora por la Universidad de Alicante. Docente en el grado de publicidad y relaciones públicas, en el ámbito de los sistemas y procesos en la empresa publicitaria y de relaciones públicas. Docente y coordinadora de prácticas en empresas en el máster en comunicación e industrias creativas. Sus principales líneas de investigación son la gestión de la comunicación en la empresa de franquicia, la comunicación de las drogas legales, comunicación para públicos específicos, y sistemas y procesos de la comunicación empresarial. Pertenece al grupo de investigación Compubes en la Universidad de Alicante y es miembro del Observatorio de Revistas Científicas de Ciencias Sociales.

http://orcid.org/0000-0002-8706-3180

mc.quiles@ua.es

Universidad de Alicante, Departamento de Comunicación y Psicología Socia Campus San Vicente del Raspeig, Ap. 99. 03080 Alicante, España

\section{Resumen}

Se realiza un análisis descriptivo de las técnicas de marketing utilizadas por las empresas de franquicia españolas y las extranjeras operativas en España. El objetivo es describir y analizar los recursos de comunicación utilizados por este tipo de empresas para proveer de información a sus tres principales públicos objetivos: las unidades operativas con que cuenta la cadena (franquiciados), los inversores dispuestos a abrir nuevas unidades (posibles franquiciados) y los clientes finales de la empresa. Se analiza la pertinencia de tales recursos desde la propia perspectiva de los empresarios franquiciadores. Los resultados apuntan a un alto uso de los medios disponibles, y en las conclusiones se plantea su interrelación desde la nueva teoría estratégica de comunicación.

\section{Palabras clave}

Franquicias; Empresas; Marketing; Comunicación; Estrategias; Sistemas de información; Encuestas.

\section{Title: Marketing information resources in franchise companies}

\begin{abstract}
A descriptive analysis of marketing techniques used by Spanish and foreign franchise companies operating in Spain has been carried out. The aim was to describe and analyse the relevance of the communication resources used by these companies when providing information to their three main targeted groups: existing units (franchisees), investors who are ready to open new units (possible franchisees), and final customers of the company. The results show high use of the communication resources and, from the viewpoint of the new strategic communication theory, the conclusion suggests that these resources should be interrelated.
\end{abstract}

\section{Keywords}

Franchising; Companies; Marketing; Communication; Strategies; Information systems; Surveys. 
Monserrat-Gauchi, Juan; Quiles-Soler, María-Carmen (2015). "Marketing de información en empresas de franquicia”. El profesional de la información, v. 24, n. 1, enero-febrero, pp. 31-38.

http://dx.doi.org/10.3145/epi.2015.ene.04

\section{Introducción}

La correcta gestión de la comunicación de forma sistémica e integral es una cuestión fundamental para el éxito de cualquier organización con independencia de su origen $u$ objeto de negocio. En el caso de la franquicia, un sistema de comercialización de bienes y servicios que goza de un auge imparable en las sociedades de consumo de los países desarrollados, es fundamental el modo en que utiliza las herramientas de marketing que tiene a su disposición. Sólo con una correcta gestión de las mismas se logra la expansión y la consecución de asociados.

Sólo con una correcta gestión de la comunicación de forma sistémica e integral se logra la expansión y la consecución de asociados

El sistema de franquicia es un modelo de negocio muy utilizado en nuestra sociedad que genera anualmente una gran cantidad de puestos de trabajo. Según el Informe de franquicia en España 2013, realizado por Tormo Franchise Consulting, en 2013 existían en España 852. Para el Registro Oficial de Franquiciadores, el número total de franquicias operativas ascendía a 1.227. En cuanto al número de establecimientos, según Tormo Franchise Consulting, había registrados un total de 51.911 en 2013, mientras que según el Registro Oficial de Franquiciadores la cifra se situaba en 45.277.

Esas altas cifras de negocio ponen de relevancia la importancia del sistema. Los resultados de investigaciones anteriores, como Bolea-de-Anta (1988), Monserrat-Gauchi (2008), Monserrat-Gauchi; Quiles-Soler; González-Díaz (2014), Argerich-Pérez (2007), Baena-Graciá; Moreno-Sánchez; Reis-Graeml (2011), demuestran que la comunicación en la empresa de franquicia se gestiona de forma diferente a las empresas convencionales y además contribuye al éxito -entendido como crecimiento- de estas empresas en el mercado. En esta investigación se estudia el modo en que la franquicia utiliza los recursos de marketing para gestionar la comunicación con sus tres grandes públicos:

- franquiciados actuales;

- franquiciados potenciales;

- consumidor final.

La comunicación es el nexo de unión entre los agentes del sistema publicitario según Mohr, Fisher y Nevin (1996). Además favorece las relaciones que se generan entre los elementos que conforman el sistema de franquicia (Anderson; Narus, 1984; Mohr; Nevin, 1990). Autores como Allen (1994), Laurie (2000) o Fernández-Monroy y Melián-Alzola (2005) afirman que la comunicación es el cauce fundamental para obtener unas relaciones óptimas en el sistema de franquicia. Por lo tanto, la identificación y el análisis de las herramientas de marketing disponibles y utilizadas nos pue-

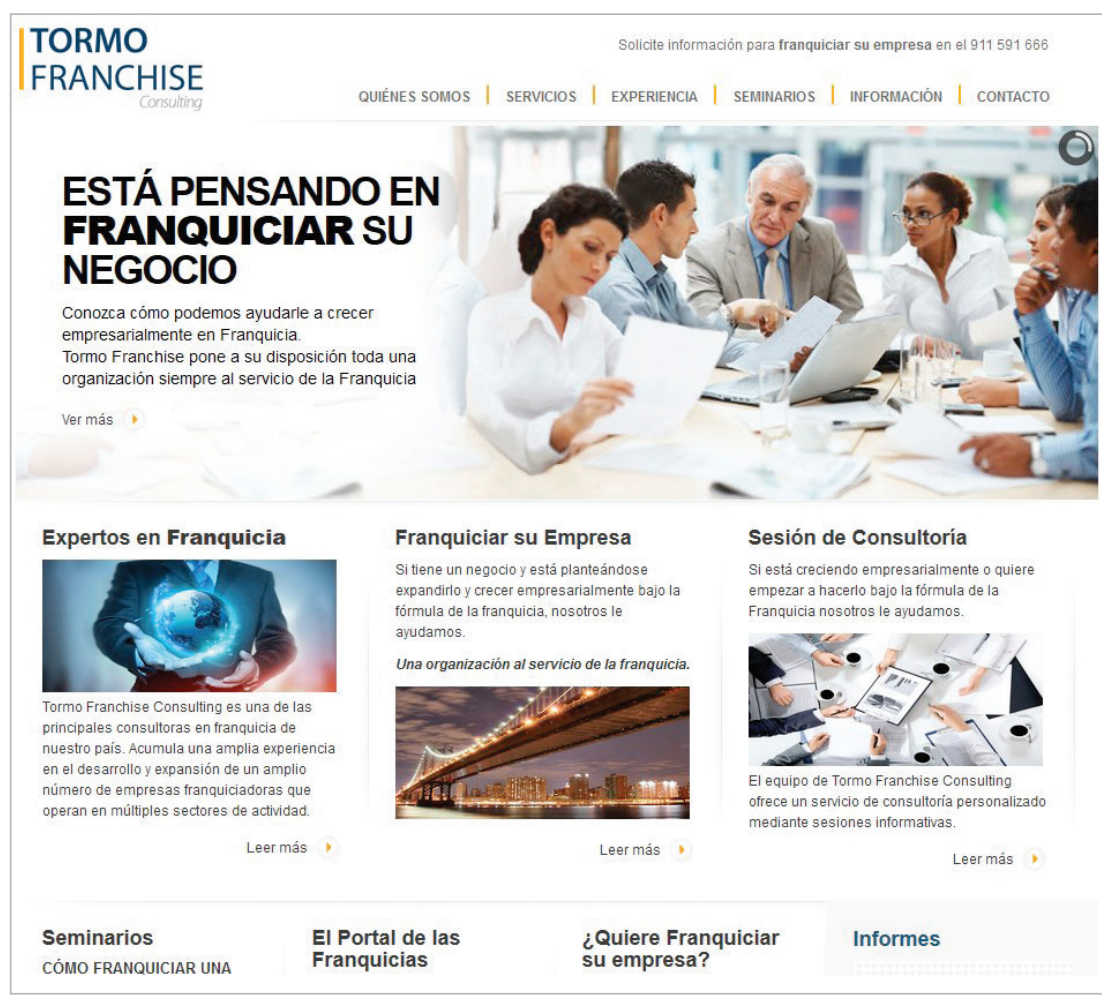

http://www.tormofranchise.com de ayudar a entender el modo en que se genera y mantiene la relación entre una franquicia y sus públicos. También es importante conocer los resultados obtenidos tras la aplicación de estos principios. Sin embargo, como se verá más adelante, el $24 \%$ de las empresas analizadas no realizan esta valoración.

Respecto al marketing y la comunicación como fenómenos de estudio abordados en la investigación, cabe destacar que han sido disciplinas estudiadas ampliamente por reconocidos investigadores. En el ámbito del marketing destacan las aportaciones de Kotler et al. (2000), Santesmases (2004) y Porter (2003; 2008), que contribuyen a delimitar y definir el ámbito de actuación de esta disciplina y su interrelación con la comunicación. Por otro lado, la comunicación ha sido analizada por autores como Van-Riel (2003) quien defiende una perspectiva integrada de comunicación de marketing en la que recoge publicidad, promociones de ventas, marketing directo, patrocinios, etc. Ar- 
genti, Howell y Beck (2005) la analizan desde un punto de vista estratégico, enmarcado en la estrategia general de la empresa. Costa (2001; 2003), Fombrun y Van-Riel (1997), así como Fombrun (2001), abordan el estudio de la comunicación desde un enfoque corporativo. Estas áreas de investigación han producido una prolífica bibliografía académica que excede los objetivos de este artículo que, sin embargo, sustenta sus bases en las clasificaciones realizadas por esos autores para analizar la comunicación de marketing (sus aplicaciones y herramientas concretas: intranet, newsletters, portales web, etc.) en la franquicia.

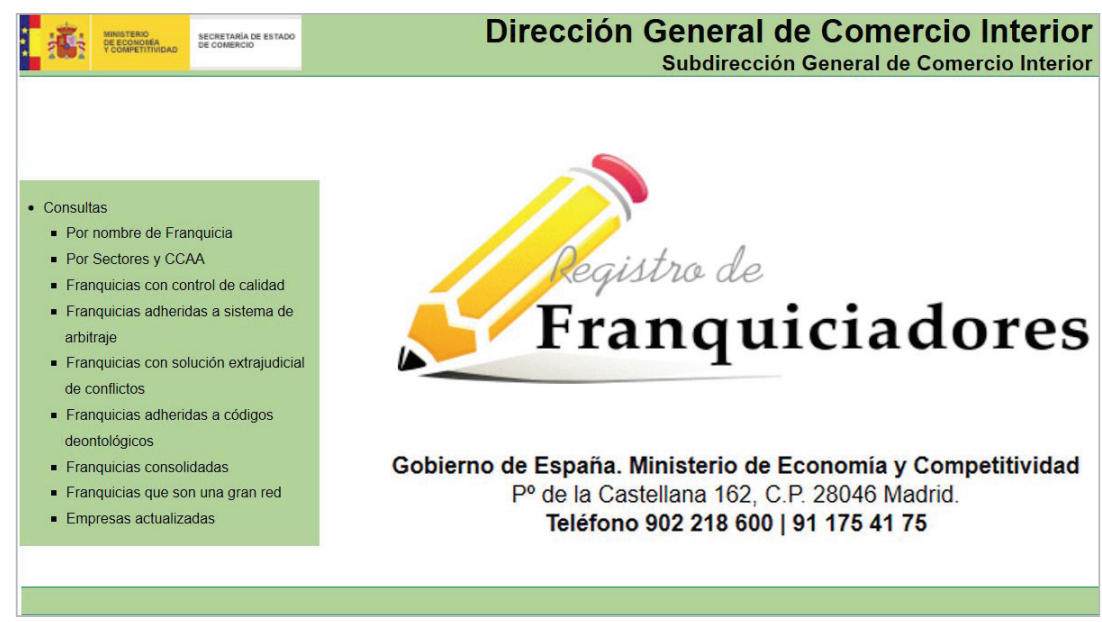

http://franquicias.comercio.es
Se acota el estudio al territorio nacional español por criterios de proximidad geográfica y accesibilidad a las empresas. Los resultados aquí expuestos son los directamente relacionados con los recursos de información en marketing y comunicación.

La comunicación es el cauce fundamental para obtener unas relaciones óptimas en el sistema de franquicia

\section{Metodología}

\section{Universo de estudio}

El universo de estudio lo forma la totalidad de las firmas o enseñas inscritas en el Registro Oficial de Franquiciadores, perteneciente al Ministerio de Economía y Competitividad del Gobierno de España en 2012. De este Registro se tomaron las empresas de la categoría Enseñas consolidadas. Estos datos se contrastaron con el registro de franquicias que ofrece Tormo \& Asociados. Tras verificar la existencia, operatividad y sistema de comercialización de las empresas, se obtuvo un universo de estudio final de 350. A todas ellas se envió el cuestionario diseñado ad-hoc para la investigación, obteniendo un nivel de respuesta del $18 \%$.

\section{Sistema de recogida de información}

Como herramienta metodológica se utilizó una encuesta estructurada online. Además de varias preguntas de control, había otras 64 agrupadas en 4 bloques:

- 20 preguntas sobre cuestiones generales de las empresas consultadas.

- 14 preguntas sobre las acciones que las enseñas llevan a cabo para sus franquiciados actuales.

- 8 preguntas para conocer cómo son las acciones que dirigen a sus franquiciados potenciales.

- 22 preguntas para identificar los recursos de marketing de información empleados para llegar al consumidor final.

Los objetivos concretos del cuestionario atienden a la identificación y medición de la eficacia en la gestión de la comunicación en este tipo de empresas, así como al análisis de la pertinencia de las estrategias utilizadas y su contribución al objetivo general de la franquicia (crecimiento y expansión).

\section{Marketing de información para las unidades operativas en franquicia}

Las empresas analizadas utilizan los siguientes recursos de marketing para la información y comunicación con sus franquiciados actuales:

\section{Boletín interno o newsletter}

El 53\% de las empresas analizadas utiliza un boletín o newsletter digital vía web o correo electrónico, el $5 \%$ en papel y el $7 \%$ ambos tipos.

El grado de efectividad del boletín percibido por los propios franquiciadores es para un $83 \%$ muy efectivo o efectivo. Sólo un $17 \%$ lo considera poco o nada efectivo.

La periodicidad del boletín es mensual en un $42 \%$ de los casos, trimestral en un $22 \%$, semanal un $8 \%$, anual un $3 \%$ y sin periodicidad fija un $8 \%$.

Diseño y ejecución de un plan de comunicación y/o promoción para sus franquiciados

\begin{tabular}{|l|r|}
\hline \multicolumn{1}{|c|}{ 24. Plan de comunicación } & $\%$ \\
\hline Sí, lo diseño y ejecuto & 55 \\
\hline Sí, asesoro y el franquiciado ejecuta & 31 \\
\hline No & 10 \\
\hline Ns/Nc & 4 \\
\hline Total general & 100 \\
\hline
\end{tabular}

El grado de efectividad del plan estratégico de comunicación percibido por los propios franquiciadores, se manifiesta así:

\begin{tabular}{|l|r|}
\hline \multicolumn{1}{|c|}{$\begin{array}{c}\text { 24.1. Efectividad percibida del plan estratégico de } \\
\text { comunicación }\end{array}$} & $\%$ \\
\hline Muy efectiva & 16 \\
\hline Efectiva & 58 \\
\hline Poco efectiva & 2 \\
\hline Ns/Nc & 24 \\
\hline Total general & 100 \\
\hline
\end{tabular}


Llama la atención que el $24 \%$ de los franquiciadores no respondan a la pregunta sobre la efectividad del plan estratégico de comunicación. Podría deberse a que no lo evalúan o llevan a cabo acciones de seguimiento; se comentará más adelante.

\section{Otros servicios de marketing}

Se consultó a los departamentos de marketing de las centrales franquiciadoras por otro tipo de servicios de marketing de información para su red de establecimientos, y éstas fueron las acciones llevadas a cabo:

\begin{tabular}{|l|c|}
\hline $\begin{array}{c}\text { 25. Acciones desarrolladas desde la central para las } \\
\text { unidades operativas }\end{array}$ & Respuestas \\
\hline Marketing directo, cartas personalizadas, etc. & 22 \\
\hline Revista o catálogo de producto & 24 \\
\hline Medios masivos (radio, tv, vallas, buses, metro) & 31 \\
\hline Marketing por internet, mensajes telefónicos, móvil, etc. & 36 \\
\hline
\end{tabular}

Respecto a la efectividad percibida por los propios franquiciadores sobre las acciones desarrolladas para los establecimientos franquiciados, se describe para cada una de las acciones:

\begin{tabular}{|l|c|c|c|c|c|}
\hline & $\begin{array}{c}\text { Muy } \\
\text { efectiva }\end{array}$ & Efectiva & $\begin{array}{c}\text { Poco } \\
\text { efectiva }\end{array}$ & $\begin{array}{c}\text { Nada } \\
\text { efectiva }\end{array}$ & $\begin{array}{c}\text { Total } \\
\text { general }\end{array}$ \\
\cline { 2 - 6 } & \multicolumn{5}{|c|}{$\%$} \\
\hline $\begin{array}{l}\text { 25.1. Marketing direc- } \\
\text { to, cartas personali- } \\
\text { zadas... }\end{array}$ & 59 & 18 & 23 & 0 & 100 \\
\hline $\begin{array}{l}\text { 25.2. Revista o catálo- } \\
\text { go de producto }\end{array}$ & 25 & 58 & 13 & 4 & 100 \\
\hline $\begin{array}{l}\text { 25.3. Medios masivos } \\
\text { 25.8. Marketing inte- }\end{array}$ & 16 & 55 & 23 & 6 & 100 \\
\hline ractivo, sms... & 28 & 47 & 25 & 0 & 100 \\
\hline
\end{tabular}

\section{Intranet}

Se consultó a las centrales de franquicia sobre la existencia en su propia empresa de una intranet, con acceso restringido a sus unidades, útil para la gestión de la red de establecimientos y también como instrumento de comunicación con sus franquiciados, con los siguientes resultados:

\begin{tabular}{|l|c|}
\hline \multicolumn{1}{|c|}{ 26. Existencia de intranet u otros medios } & Respuestas \\
\hline Sí, poseo intranet & 39 \\
\hline $\begin{array}{l}\text { Utilizo otros medios como Facebook, redes sociales en } \\
\text { internet, Skype o Messenger, etc. }\end{array}$ & 34 \\
\hline No & 6 \\
\hline
\end{tabular}

La mayoría de las empresas que poseen intranet la consideran muy efectiva o efectiva (95\%).

Las empresas que utilizan otros medios, como los descritos, perciben su efectividad en base a los siguientes datos: muy efectivos el $29 \%$, efectivos el $53 \%$, y poco efectivos el $18 \%$.

Entre las utilidades de la intranet como instrumento de marketing para la transmisión de información a las unidades operativas de la empresa, los directores de marketing destacan las siguientes:

\begin{tabular}{|l|c|}
\hline \multicolumn{1}{|c|}{ 28. Utilidades de la intranet } & $\%$ \\
\hline Pagos & 29 \\
\hline Comunicación entre franquiciados & 42 \\
\hline Sugerencias de los franquiciados & 49 \\
\hline Pedidos & 47 \\
\hline $\begin{array}{l}\text { Página informativa para el franquiciado, con repositorio de } \\
\text { documentos de interés }\end{array}$ & 65 \\
\hline Información de nuevos productos o servicios & 67 \\
\hline
\end{tabular}

La comunicación entre las unidades operativas (42\%), la posibilidad de realizar sugerencias a la central por parte de éstas (49\%), el hecho de que la intranet sea una página informativa para los franquiciados en la que se les puedan proporcionar documentos e información de interés (65\%), además de información sobre nuevos productos y servicios (67\%), convierten a este instrumento en uno de los más implantados.

\section{Marketing de información para la captación de franquiciados}

Otro de los públicos de interés para este tipo de organizaciones son los posibles franquiciados. Éstos son normalmente personas en búsqueda activa de empleo, o bien inversores que no van a estar al frente del negocio pero buscan obtener beneficios.

Los directores de marketing utilizan los siguientes materiales de promoción en sus empresas:

\section{Campaña de publicidad}

Un 63\% de las empresas analizadas realiza campañas de publicidad pagadas, exclusivamente dirigidas a la captación de franquiciados; un $35 \%$ no realiza, y un $2 \%$ no respondió.

La valoración de la efectividad de estas campañas es alta, en tanto que se consideró muy efectiva o efectiva por un $74 \%$, poco efectiva por el $12 \%$, nada efectiva por un $6 \%$ y un $8 \%$ no contestó a esta pregunta.

\section{Instrumentos de marketing}

Los instrumentos de marketing y medios publicitarios utilizados por las empresas en estas campañas de captación son los siguientes:

\begin{tabular}{|l|c|}
\hline \multicolumn{1}{|c|}{ 36. Medios para la captación de franquiciados } & Respuestas \\
\hline $\begin{array}{l}\text { Portales web de periódicos online: elpais.com, elmundo. } \\
\text { es, etc. }\end{array}$ & 10 \\
\hline $\begin{array}{l}\text { Medios especializados en el sector de actividad de la } \\
\text { empresa (alimentación, restauración, decoración, etc.) }\end{array}$ & 15 \\
\hline $\begin{array}{l}\text { Prensa, general o económica (periódicos, suplementos } \\
\text { salmón o diarios económicos) }\end{array}$ & 16 \\
\hline Redes sociales profesionales & 16 \\
\hline $\begin{array}{l}\text { Acciones de marketing directo (presentaciones perso- } \\
\text { nalizadas, cartas, utilización de bases de datos, etc.) }\end{array}$ & 21 \\
\hline Asistencia a ferias de franquicia (SIF, Expofranquicia, etc.) & 23 \\
\hline $\begin{array}{l}\text { Prensa específica de franquicias, revistas, anuarios, guías } \\
\text { de franquicia }\end{array}$ & 29 \\
\hline $\begin{array}{l}\text { Portales web de consultoras en franquicia (tormo.com, } \\
\text { mundofranquicia.com, etc.) }\end{array}$ & 41 \\
\hline
\end{tabular}




\section{Web de la empresa}

Otro canal importante es la web de la propia empresa, como escaparate en el que proporcionar información sobre el posible negocio, rentabilidad del mismo, etc. Lo utiliza un $87 \%$ de ellas y es muy valorada (el $84 \%$ la considera muy efectiva o efectiva) para la captación de nuevos asociados a la red de franquicia. Se consultó a los directores de marketing de las empresas sobre los elementos disponibles en dicha web:

\begin{tabular}{|l|c|c|}
\hline \multirow{2}{*}{$\begin{array}{c}\text { 39. Recursos de información de marketing } \\
\text { disponibles en la web }\end{array}$} & Sí & No \\
\cline { 2 - 3 } & \multicolumn{2}{|c|}{$\%$} \\
\hline Formulario de solicitud de información & 87 & 13 \\
\hline Catálogo o dossier informativo de la franquicia & 66 & 34 \\
\hline Información legal & 65 & 35 \\
\hline
\end{tabular}

Y su efectividad:

\begin{tabular}{|l|c|c|c|c|c|}
\hline $\begin{array}{c}\text { 39.1/2/3. Grado de } \\
\text { efectividad }\end{array}$ & $\begin{array}{c}\text { Muy } \\
\text { efectivo }\end{array}$ & Efectivo & $\begin{array}{c}\text { Poco } \\
\text { efectivo }\end{array}$ & $\begin{array}{c}\text { Nada } \\
\text { efectivo }\end{array}$ & Ns/Nc \\
\cline { 2 - 6 } & \multicolumn{5}{|c|}{$\%$} \\
\hline $\begin{array}{l}\text { Formulario de solicitud } \\
\text { de información }\end{array}$ & 38 & 42 & 10 & 2 & 8 \\
\hline $\begin{array}{l}\text { Catálogo o dossier infor- } \\
\text { mativo de la franquicia }\end{array}$ & 17 & 67 & 6 & 3 & 7 \\
\hline Información legal & 10 & 61 & 16 & 3 & 10 \\
\hline
\end{tabular}

\section{Marketing de información para el cliente}

El tercer público de interés para este tipo de empresas, no menos importante, es el cliente final. Para este público, los directores de marketing también planifican promociones, publicidad y utilizan técnicas de marketing tanto para ofrecer información sobre la empresa, como para recoger información de los clientes. A continuación se detallan las principales acciones de marketing de información llevadas a cabo.

\section{El $24 \%$ de los franquiciadores no respon-} de a la pregunta sobre la efectividad del plan estratégico de comunicación, ¿̇uede deberse a que no lo evalúan?

\section{Campaña de marketing promocional}

Se preguntó si desde la central de franquicia se planifica y ejecuta una campaña de marketing promocional para la apertura de cada establecimiento franquiciado, y un altísimo porcentaje respondió que sí (el 89\%), por un 9\% que no y un $2 \%$ no respondió.

Con respecto a la percepción sobre su efectividad, el $88 \%$ la perciben efectiva o muy efectiva, el $8 \%$ poco o nada efectiva y un $4 \%$ no responde a esta cuestión.

\section{Promociones}

Otra de las acciones de marketing de información para los clientes finales son las promociones. Se preguntó a los directores si, en caso de que desde el departamento de marketing se planifiquen promociones para los establecimientos operativos de la cadena, éstas se reflejan en la web. Las respuestas en este caso están más equilibradas: el 52\% respondió que sí se reflejan en la web, el $37 \%$ que no, y el $11 \%$ no respondió.

Además se pidió que valorasen el grado de efectividad de las mismas, indicando un $77 \%$ que son efectivas o muy efectivas, un $19 \%$ que son poco o nada efectivas y un $4 \%$ no respondió a esta pregunta.

\section{Datos de los clientes}

El siguiente aspecto a analizar fue si las empresas recogen los datos de sus clientes (teléfono móvil, correo electrónico, etc.) con la finalidad de poseer formas de contacto directo con ellos para informarles de promociones, acciones de marketing directo, u ofertas puntuales. Un alto porcentaje sí lo hace (el $78 \%$ ), por un $18 \%$ que no, y un $4 \%$ no respondió.

La medición de la efectividad de esta pregunta nos muestra un $72 \%$ de empresas que lo valora positivamente, un $11 \%$ con una efectividad poca o nula y un $16 \%$ no muestra su valoración al respecto.

\section{Contenido de la web de la franquicia}

Dado que la mayoría de empresas de franquicia (85\%) posee una web destinada a los clientes finales de sus establecimientos franquiciados, se ha considerado interesante analizar el contenido de la misma. Los directores de marketing manifiestan que poseen los siguientes elementos de información:

\begin{tabular}{|l|c|}
\hline \multicolumn{1}{|c|}{ 55. Contenido de la web } & $\%$ \\
\hline Foro de discusión entre clientes & 4 \\
\hline $\begin{array}{l}\text { Apartados de valor añadido (juegos, interactividad, informa- } \\
\text { ción) para el visitante de la web }\end{array}$ & 15 \\
\hline Atención online en tiempo real & 16 \\
\hline Visita virtual & 16 \\
\hline Apartado "club" socios/clientes & 18 \\
\hline Sugerencias y reclamaciones & 29 \\
\hline Información del sector & 29 \\
\hline Suscripción a newsletter & 29 \\
\hline Sala de prensa & 42 \\
\hline Noticias dirigidas al consumidor & 49 \\
\hline Ofertas especiales & 51 \\
\hline Catálogo de productos & 67 \\
\hline Solicitud de información & 82 \\
\hline Listado de establecimientos & 84 \\
\hline Contacto & 89 \\
\hline
\end{tabular}

Se consideró necesario conocer la frecuencia de actualización de los contenidos de dicha web, y éstas son las respuestas:

\begin{tabular}{|l|c|}
\hline 56. Frecuencia de actualización de la web & \% \\
\hline Diaria & 29 \\
\hline Semanal & 31 \\
\hline Mensual & 24 \\
\hline Trimestral & 7 \\
\hline Semestral & 7 \\
\hline Anual & 0 \\
\hline Ns/Nc & 2 \\
\hline Total & 100 \\
\hline
\end{tabular}




\section{Presencia en redes sociales}

Además de la web, se preguntó a los directores de las empresas de franquicia sobre su presencia en redes sociales y el uso que realizan de las mismas como instrumento de información de marketing, con los siguientes resultados:

\begin{tabular}{|l|c|}
\hline 61. Presencia y uso de canales $\mathbf{2 . 0}$ & \% \\
\hline Facebook & 89 \\
\hline Twitter & 75 \\
\hline YouTube & 64 \\
\hline Blog & 36 \\
\hline Foursquare & 16 \\
\hline Otros & 22 \\
\hline Ninguno & 5 \\
\hline
\end{tabular}

Respecto al principal objetivo de la presencia redes sociales, manifestaron:

\begin{tabular}{|l|c|}
\hline \multicolumn{1}{|c|}{ 62. Objetivo de la presencia en redes sociales } & \% \\
\hline Investigación de mercado & 29 \\
\hline Comunicación personalizada & 31 \\
\hline Conversión a ventas & 36 \\
\hline Servicio y atención al cliente & 55 \\
\hline Generación de comunidad & 58 \\
\hline Construcción de marca & 75 \\
\hline Otros & 0 \\
\hline Ns/Nc & 0 \\
\hline
\end{tabular}

\section{Acciones de marketing online}

Por último se consideró relevante conocer qué acciones de marketing online se han llevado a cabo por parte de las empresas de franquicia, con los siguientes resultados:

\begin{tabular}{|l|c|}
\hline \multicolumn{1}{|c|}{ 64. Acciones de marketing online } & Respuestas \\
\hline Marketing de afiliación & 12 \\
\hline Marketing a través de móvil & 13 \\
\hline Comercio electrónico & 15 \\
\hline SEM (marketing en buscadores) & 23 \\
\hline Displays (publicidad online) & 26 \\
\hline SEO & 31 \\
\hline Marketing por correo-e & 38 \\
\hline Redes sociales & 44 \\
\hline Otros & 0 \\
\hline
\end{tabular}

\section{Conclusiones y discusión}

En el ámbito de la teoría estratégica es reseñable la aportación de Pérez-González y Massoni (2009). Ambos inciden en la necesidad de formular una nueva teoría estratégica con la que los autores de esta investigación están en sintonía, en cuanto a que supone un cambio de paradigma que afecta a la concepción de la estrategia en sí y a su gestión y aplicación en el entorno empresarial.

El paradigma de la nueva teoría estratégica (NTE) pasa de lo económico a la complejidad de públicos y relaciones en la organización, asumiendo la no linealidad y el caos como características de los mercados o sociedades actuales. Tiene una visión holística y no reduccionista de la organización, y más que pretender una fragmentación analítica realiza un análisis sistémico. Desde esta perspectiva se debería llevar a cabo el estudio de la gestión de las estrategias de comunicación en la franquicia.

En esta línea se ha contemplado el presente estudio atendiendo a la posibilidad de una comunicación fluida y bidireccional. Es necesario gestionar la estrategia de comunicación en base a los postulados de la NTE, en la que un nuevo modelo de interacción en la organización tenga en cuenta los

\section{Se percibe una conciencia manifiesta de la importancia de la gestión estratégica de la comunicación en estas empresas}

sucesos emergentes que forman parte de cualquier sistema abierto. Que permita generar acciones basadas en la gestión de realidades, más que en la persuasión de los receptores.

De acuerdo con Anderson y Weitz (1989), la franquicia responde a un modelo de negocio que exige una muy buena relación entre las partes, y la correcta gestión de la comunicación y la confianza entre franquiciador y franquiciado son dos variables indispensables para evitar conflictos y consolidar relaciones. Algunos estudios han demostrado que existen ciertos aspectos directamente relacionados con la comunicación franquiciador-franquiciado que satisfacen a éste, como puede ser el uso de marcas reconocidas o el envío de boletines de información y manuales de franquicia (Knight, 1986).

Son muchos los autores (Allen, 1994; Mubleman, 1996; Laurie, 2000; Tikoo, 2002; Fernández-Monroy; Melián-Alzola, 2005) que afirman que la comunicación es la vía para conseguir una buena relación en el sistema de franquicia. Siguiendo con esta afirmación, pretendemos contribuir a un modelo de gestión estratégica que conciba la gestión de la comunicación atendiendo a los públicos ya definidos y además a los cambios tecnológicos y económicos en los que las empresas se desenvuelven.

\section{Se hace necesaria una reflexión sobre la necesidad de implantar en mayor grado los postulados de las nuevas teorías es- tratégicas}

Del análisis descriptivo llevado a cabo, cabría destacar algunos aspectos referidos a la gestión de los recursos de marketing de información en las empresas españolas y extranjeras operativas en España. Si bien se detecta un alto uso de los recursos disponibles en este tipo de empresas hay algunos factores que cabría comentar:

a) Se percibe una conciencia manifiesta respecto a la importancia de la gestión estratégica de la comunicación en estas empresas. El 86\% de las mismas diseñan y ejecutan, o bien 
asesoran para que sus franquiciados ejecuten, un plan estratégico de comunicación (PEC). Por otro lado y en sentido opuesto, el $24 \%$ no valora la eficacia de este plan. La última etapa de un PEC y una de las más importantes es su evaluación (Monserrat-Gauchi, 2014).

b) El uso de las nuevas tecnologías y de los recursos online es notable. Las empresas optimizan el uso de internet (y de la intranet) para dirigirse a sus diferentes públicos, pero llama la atención que el $24 \%$ actualice los contenidos de sus webs sólo mensualmente. Destaca el uso concedido a la web propia y a las de consultoras para la captación de nuevos franquiciados, así como el número de contenidos de marketing de información recogidos en la mayoría de las webs.

c) Los autores de esta investigación consideran que la franquicia utiliza correctamente los sistemas de marketing para la información a sus públicos. No obstante, se hace necesaria una reflexión sobre la necesidad de implantar en mayor grado los postulados de las nuevas teorías estratégicas en comunicación en la empresa de franquicia.

\section{Gestionar la estrategia de comunicación} en base a los postulados de la NTE supone crear una comunicación fluida y bidireccional

\section{Nota}

Los resultados aquí presentados forman parte de una investigación financiada por la Universidad de Alicante, con referencia: GRE11-16.

\section{Bibliografía}

Allen, Robin-Lee (1994). "Franchisor-franchisee: communication maintains the marriage". Nation's restaurant news, v. 28, n. 12, p. 27.

Anderson, Erin; Weitz, Barton (1989). “Determinants of continuity in conventional industrial channel dyads". Marketing science, v. 8, n. 4, pp. 310-323.

http://bear.warrington.ufl.edu/weitz/papers/Determinants Continuity.pdf

Anderson, James C.; Narus, James A. (1984). "A model of the distributor's perspective of distributor-manufacturer working relationships". Journal of marketing, v. 48, pp. 62-74.

http://www.jstor.org/stable/1251511

http://dx.doi.org/10.2307/1251511

Argenti, Paul A.; Howell, Robert A.; Beck, Karen A. (2005). "The strategic communication imperative". MIT Sloan management review, v. 46, n. 3, pp. 82-89.

http://cimsec.org/wp-content/uploads/2014/11/ SloanMITStratCommImp.pdf

Argerich-Pérez, Javier (2007). Comunicación y franquicia: la comunicación como herramienta de gestión en las franquicias españolas. Madrid: Ed. Fragua. ISBN: 9788470742323

Armstrong, Gary; Kotler, Philip (2012). Marketing: an introduction. New Jersey, Prentice- Hall. ISBN: 9780132744034
Baena-Graciá, Verónica; Moreno-Sánchez, María-de-Fátima; Reis-Graeml, Felipe (2011). "Las ferias comerciales como herramienta de comunicación y generadora de valor de una compañía. Análisis de la franquicia española". Tribuna de economía, n. 860, pp. 147-162.

http://www.revistasice.com/CachePDF/ICE_860_147162_51D646931F227A73396CC438D06601E9.pdf

Bolea-de-Anta, Adelaida (1988). La empresa de franquicia en el ámbito de la comunicación: Un modelo de aplicación al campo del diseño y moda de España. Tesis doctoral inédita. Madrid: Universidad Complutense de Madrid.

Costa, Joan (2001). Imagen corporativa en el siglo XXI. Buenos Aires: La Crujía. ISBN: 9789879749890

Costa, Joan (2003). “Creación de la imagen corporativa. El paradigma del siglo XXI". Razón y palabra, n. 34.

http://www.razonypalabra.org.mx/anteriores/n34/jcosta.html

Fernández-Monroy, Margarita; Melián-Alzola, Lucía (2005). "An analysis of quality management in franchise system". European journal of marketing, v. 39, n. 5/6, pp. 585-605. http://dx.doi.org/10.1108/03090560510590728

Fombrun, Charles (2001). "Corporate reputation as economic assets". En: Hitt, Michael; Freeman, Edward; Harrison, Jeffrey. The Blackwell handbook of strategic management. USA: Blackwell. ISBN: 9780631218616 http://dx.doi.org/10.1111/b.9780631218616.2006.x

Fombrun, Charles; Van-Riel, Cees (1997). "The reputational landscape". Corporate reputation review, v. 1, n. 1, 1997, pp.5-13

http://repub.eur.nl/pub/12136/TheReputationalLandscape_1997. $p d f$

Knight, Russell M. (1986). "Franchising from the franchisor and franchisee points of view". Journal of small business management, v. 24, pp. 8-15.

Kotler, Philip; Cámara, Dionisio; Grande, Ildefonso; Cruz, Ignacio (2000). Dirección de marketing. Madrid: PrenticeHall. ISBN: 9788483222089

Laurie, Crystal (2000). "Partners for the millennium". Franchising world, n. 32, pp. 12-20.

Mohr, Jakki J.; Fisher, Robert J.; Nevin, John R. (1996). "Collaborative communication in interfirm relationships: Moderating effects of integration and control". Journal of marketing, v. 60, n. 3, pp. 103-115.

http://www.jstor.org/stable/1251844

http://dx.doi.org/10.2307/1251844

Mohr, Jakki J.; Nevin, John R. (1990). "Communication strategies in marketing channels: A theoretical perspective". Journal of marketing, v. 54, n. 4, pp. 36-51.

http://www.jstor.org/stable/1251758

http://dx.doi.org/10.2307/1251758

Monserrat-Gauchi, Juan (2008). "Análisis comparativo de las estrategias de comunicación en franquicia. Enseñas consolidadas versus emergentes". Revista latina de comunicación social, n. 63, pp. 106-120.

http://www.revistalatinacs.org/_2008/09_Alicante/Juan Monserrat.html 
http://dx.doi.org/10.4185/RLCS-63-2008-756-106-120

Monserrat-Gauchi, Juan (2014). Cómo elaborar un plan estratégico de comunicación. Alicante: Publicaciones de la Universidad de Alicante. ISBN: 9788497173223

Monserrat-Gauchi, Juan; Quiles-Soler, María-Carmen; González-Díaz, Cristina (2014). "La innovación en las estrategias para la captación de franquiciados". Palabra clave, v. 17, n. 2, pp. 517-545.

http://palabraclave.unisabana.edu.co/index.php/ palabraclave/article/view/3018/3508

Mubleman, Janet (1996). "The franchise relationship channel". Franchising world, v. 28, n. 1, pp. 20-24.

Pérez-González, Rafael-Alberto; Massoni, Sandra (2009). Hacia una teoría general de la estrategia. Barcelona: Ariel. ISBN: 9788434413108

Porter, Michael E. (2003). Ser competitivo, nuevas aportaciones y conclusiones. Bilbao: Ed. Deusto, ISBN: 978 8423421138

Porter, Michael E. (2008). "The five competitive forces that shape strategy". Harvard business review, v. 86, n. 1, pp. 25-40. http://www.exed.hbs.edu/assets/documents/hbr-shapestrategy.pdf

Registro Oficial de Franquiciadores. Ministerio de Economía y Competitividad. Gobierno de España.

http://franquicias.comercio.es

Santesmases, Miguel (2004). Marketing: conceptos y estrategias. Madrid: Pirámide. ISBN: 8436818709

Tikoo, Surinder (2002). "Franchiser influence strategy use and franchisee dependence and experience". Journal of retailing, v. 78, n. 3, pp. 183-192.

http://dx.doi.org/10.1016/S0022-4359(02)00064-7

Tormo Franchise Consulting (2013). Informe de franquicia en España 2013.

http://www.tormofranchise.com/pdfs/informe_ franquicia_2013.pdf

Van-Riel, Cees (2003). “Nuevas formas de comunicación organizacional". Razón y palabra, n. 34.

http://www.razonypalabra.org.mx/anteriores/n34/ cvanriel.html

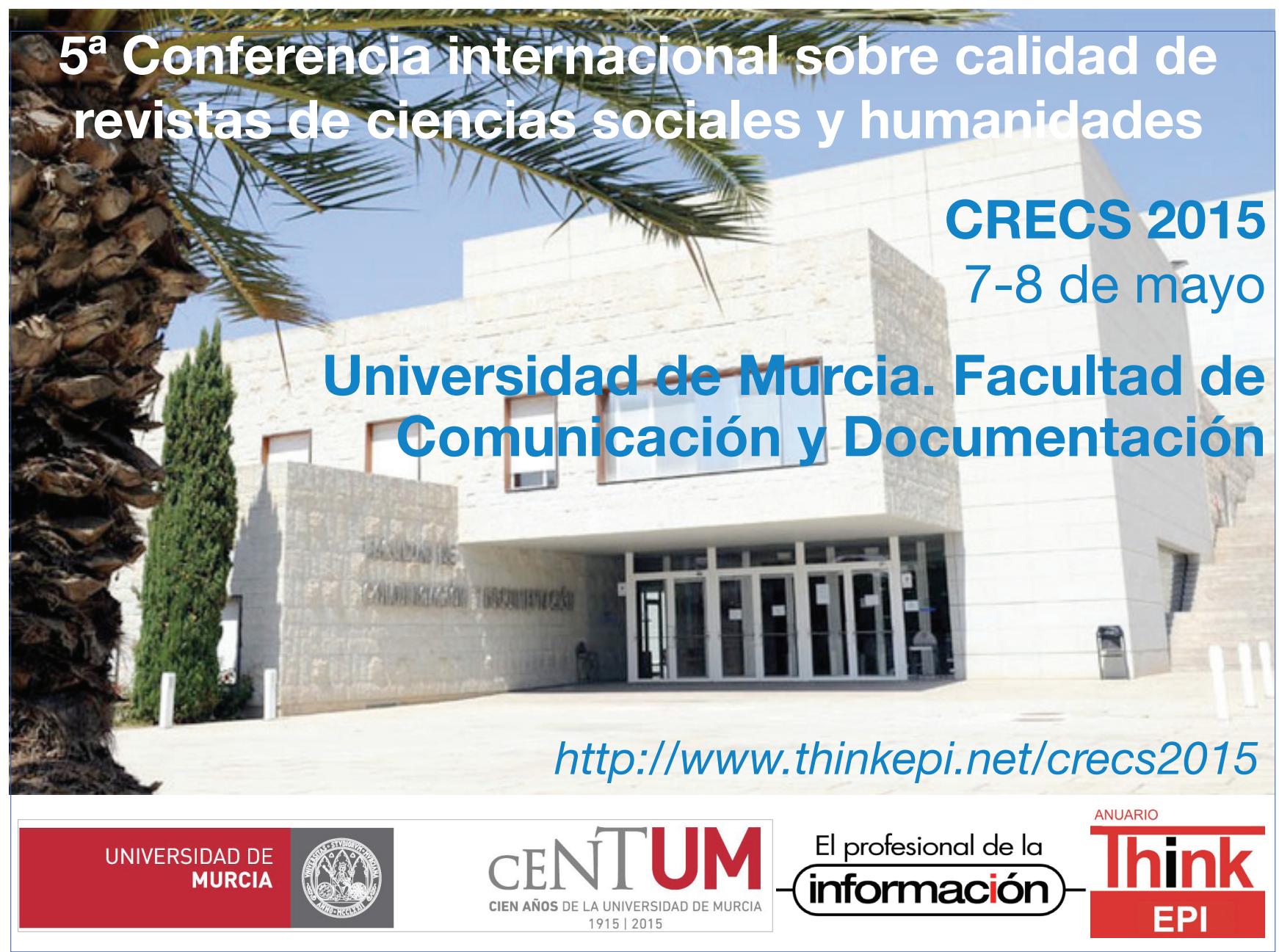

